\title{
Lactoferrin inhibits apoptosis through insulin-like growth factor I in primary rat osteoblasts
}

\author{
Jian-ming HOU ${ }^{1,2, \#, *}$, En-yu CHEN", ${ }^{\#,}$ Shi-chao WEI ${ }^{1,2, \#}$, Fan $\operatorname{LIN}^{1,2, \#}$, Qing-ming $\operatorname{LIN}^{1,2}$, Xu-hua LAN ${ }^{1,2}$, Ying XUE ${ }^{1,2}$, \\ Man $\mathrm{WU}^{2}$ \\ ${ }^{1}$ Fujian Provincial Hospital, Fuzhou 350001, China; ${ }^{2}$ Provincial Clinical Medical College of Fujian Medical University, Fuzhou 350004, \\ China
}

\begin{abstract}
Aim: Excessive apoptosis of osteoblasts is the major cause of low bone mass, and bovine lactoferrin (bLF), an iron-binding glycoprotein, might protect osteoblastic cells from apoptosis induced by serum withdrawal. The aim of this study was to elucidate the mechanisms underlying the anti-apoptotic action of bLF in rat osteoblasts in vitro.

Methods: Primary rat osteoblasts were incubated in the presence of varying concentrations of bLF for $24 \mathrm{~h}$. The expression of insulinlike growth factor I (IGF-I) and IGF-I receptor (IGF-IR) was measured uisng RT-PCR and Western blotting. Cell apoptosis was examined with flow cytometry. siRNAs targeting IGF-I was used in this study.

Results: Treatment of bLF $(0.1-1000 \mu \mathrm{g} / \mathrm{mL})$ dose-dependently increased the expression of IGF-I and IGF-IR in the osteoblasts. Treatment with bLF $(10,100 \mu \mathrm{g} / \mathrm{mL})$ markedly inhibited the osteoblast apoptosis (with the rate of total apoptosis of $70 \%$ at $10 \mu \mathrm{g} / \mathrm{mL})$, but the high concentration of bLF $(1000 \mu \mathrm{g} / \mathrm{mL})$ significantly promoted the osteoblast apoptosis. Knockdown of the IGF-I gene in osteoblasts with siRNA markedly increased the osteoblast apoptosis.

Conclusion: Lactoferrin (10 and $100 \mu \mathrm{g} / \mathrm{mL}$ ) effectively inhibits apoptosis of primary rat osteoblasts by upregulating IGF-I expression.
\end{abstract}

Keywords: lactoferrin; osteoblast; IGF-I; apoptosis; RNA interference; bone remodeling; osteoporosis

Acta Pharmacologica Sinica (2014) 35: 523-530; doi: 10.1038/aps.2013.173; published online 24 Feb 2014

\section{Introduction}

Bovine lactoferrin (bLF) is an iron-binding glycoprotein that belongs to the transferrin family and is produced by epithelial cells of the exocrine glands ${ }^{[1,2]}$. bLF can stimulate the proliferation and differentiation of bone-forming osteoblasts and act as a survival factor for these cells ${ }^{[3]}$. bLF has been demonstrated to have potent anti-apoptotic effects on osteoblasts that may also contribute to the anabolic skeletal actions of these cells ${ }^{[4,5]}$.

A healthy skeleton is maintained by the continuous renewal of bone marrow. Bone remodeling depends on the actions of two main bone cells: osteoclasts and osteoblasts. Osteoclasts resorb old bone while osteoblasts form new bone by first laying down an unmineralized matrix and then orchestrating its mineralization ${ }^{[6]}$. The excessive apoptosis of osteoblasts is the major cause of low bone mass. In addition, bLF might protect osteoblastic cells from apoptosis induced by serum withdrawal ${ }^{[4]}$. Understanding the molecular mechanisms

\footnotetext{
\#These authors contributed equally to this work.

* To whom correspondence should be addressed.

E-mail hjm996@126.com

Received 2013-08-01 Accepted 2013-11-05
}

regulating osteoblast survival may help us to develop effective therapeutics for the treatment of low bone mass in patients afflicted with diseases such as osteoporosis, primary hyperparathyroidism and systemic sclerosis.

Our previous study found that bLF at 1 and $2 \mathrm{mg} \cdot \mathrm{kg}^{-1} \cdot \mathrm{d}^{-1}$ significantly increased the bone mass and improved bone microarchitecture in ovariectomized rats suffering from increased bone loss, severe osteopenia and increased bone resorption $^{[7]}$. Previous studies have shown anabolic effects of bLF on osteoblasts and osteoclasts in vitro ${ }^{[4,8,9]}$. Grey found that bLF inhibited $50 \%-70 \%$ of apoptosis in osteoblasts, but he also discovered that this inhibition did not involve the PI3 kinase or p42/44 MAP kinase signaling pathways ${ }^{[4]}$. So the exact mechanisms for the effect of bLF on osteoblasts are largely unknown, the aim of this study was to explore the mechanisms of action of bLF on apoptosis of primary rat osteoblasts by studying the insulin-like growth factor (IGF) signaling pathway.

\section{Materials and methods Reagents}

Bovine lactoferrin was obtained from Fonterra (Auckland, 
New Zealand). Fetal bovine serum (FBS), L-glutamine, penicillin/streptomycin and D-minimal essential medium (DMEM) were obtained from Gibco-BRL (Grand Island, NY, USA). Trypsin was purchased from Hyclone (Auckland, New Zealand). The Annexin-V/PI Apoptosis kit was purchased from Sigma (America). The Reverse Transcription System kit and real-time PCR kit were purchased from Takara (Japan). The alkaline phosphatase staining kit was purchased from JianCheng Biotech Companies (China). The SABC immunohistochemistry kit was purchased from Wuhan Boster Companies (China). The Type-I Collagen Enzyme and TRIZOL were purchased from Invitrogen (Carlsbad, CA, USA). The monoclonal IGF-I antibody and the monoclonal IGF-IR antibody used for Western blot analyses were purchased from Abcam (Cambridge, UK).

\section{Cell culture}

Primary osteoblasts were isolated from the calvaria of newborn $(<24 \mathrm{~h})$ Sprague Dawley rats. Briefly, neonatal rat calvariae were dissected from adherent soft tissue and washed in a PBS solution $(2 \mathrm{~mL})$. The tissue was shredded into 1 $\mathrm{mm} \times 1 \mathrm{~mm} \times 1 \mathrm{~mm}$ pieces using ophthalmic scissors and then sequentially digested with $3 \mathrm{~mL}$ trypsin $(15 \mathrm{~min})$ followed by $4 \mathrm{~mL}$ collagenase mixed with $1 \mathrm{~mL}$ trypsin $(60 \mathrm{~min})$ to release the cells. The cell preparations were washed and cultured in D-minimum essential medium (DMEM) containing 10\% FBS and antibiotics. Cells were plated in $25-\mathrm{cm}^{2}$ flasks and cultured overnight in an incubator with a $\mathrm{CO}_{2}$ level of $5 \%$ at $37^{\circ} \mathrm{C}$. The medium was changed every other day. Cells in the third passage were used for all experiments.

\section{Identification of primary cultured rat osteoblasts Cell morphology}

Osteoblast morphology was examined using an inverted microscope.

\section{Alkaline phosphatase staining}

Third passage cells were trypsinized and seeded onto chamber slides. Alkaline phosphatase staining was performed according to the manufacturer's protocol: the cells were stained with hematoxylin for $3 \mathrm{~min}$, washed in water, and then examined by microscopy.

\section{SABC Immunohistochemistry}

SABC immunostaining was performed according to the manu- facturer's protocol with a reaction time between 5 and $30 \mathrm{~min}$. The samples were washed in water and examined by microscopy.

\section{Annexin V-FITC and propidium iodide double-stained flow cytometry}

After treatment with different concentrations of bLF $(0,0.1$, $1,10,100$, and $1000 \mu \mathrm{g} / \mathrm{mL}$ ) for $24 \mathrm{~h}$, cells were detached and rinsed with PBS to prevent aggregation. Cells were collected and stained using the Annexin V-FITC Apoptosis Detection Kit at room temperature in the dark for $10 \mathrm{~min}$ and then filtered before analysis. Cells $\left(1 \times 10^{6}\right)$ were analyzed by flow cytometry to detect and quantify cells undergoing apoptosis or necrosis. The data were analyzed using CellQuest Software.

\section{RNA isolation and real-time PCR}

To determine the expression levels of IGF-I and IGF-IR mRNAs in primary rat osteoblasts incubated with varying concentrations of $\operatorname{bLF}(0,0.1,1,10,100$, and $1000 \mu \mathrm{g} / \mathrm{mL})$ for $24 \mathrm{~h}$, total RNA was extracted using the TRIzol reagent according to the manufacturer's protocol. The concentration and purity of total RNA were calculated by measuring the absorbance at 260 and $280 \mathrm{~nm}$. Total RNA $(1 \mu \mathrm{g})$ was used for the synthesis of first strand cDNA (cDNA synthesis kit; Takara, Japan). PCR primers (Table 1) were designed using the Primer 5.0 software. RT-PCR was performed using a Thermal Cycler Dice ${ }^{\mathrm{TM}}$ RealTime System (TP800, TaKaRa). Real-time PCR was performed using $2 \mu \mathrm{L}$ of cDNA in a $25 \mu \mathrm{L}$ reaction volume that included the SYBR PremixEx Taq II (2×) (DRR081A, TaKaRa, Japan) and diluted gene specific primers. All reactions were performed in triplicate and analyzed by the $2^{-\Delta \Delta \mathrm{CT}}$ method. $\beta$-Actin was used as an internal control.

\section{Western blotting}

For Western blot analyses, osteoblasts were treated with different concentrations of $\operatorname{bLF}(0,0.1,1,10,100$, and $1000 \mu \mathrm{g} / \mathrm{mL})$ for $24 \mathrm{~h}$. Cells were washed twice with ice-cold PBS and then resuspended in lysis buffer (RIPA, Beyone, China) containing 1\% NP-40, 0.1\% SDS, $5 \mathrm{mmol} / \mathrm{L}$ EDTA, 0.5\% sodium deoxycholate, $1 \mathrm{mmol} / \mathrm{L}$ sodium orthovanadate, and $1 \mathrm{mmol} / \mathrm{L}$ phenylmethylsulfonyl fluoride. Protein concentrations were determined using a BCA protein assay (Beyotime Biotech Companies, Haimen, China). For each sample, $30 \mu \mathrm{g}$ of protein was run on a $15 \%$ polyacrylamide gel and transferred to a polyvinylidene difluoride (PVDF) membrane (Millipore,

Table 1. The primer sequences for real-time PCR.

\begin{tabular}{clll}
\hline Genes & GenBank numbers & \multicolumn{1}{c}{ Primer sequence } & Product size (bp) \\
\hline \multirow{2}{*}{-Actin } & NM 031144.2 & $\begin{array}{l}\text { Forward 5'-GGAGATTACTGCCCTGGCTCTA-3' } \\
\text { Reverse 5'-GACTCATCGTACTCCTGCTTGCTG-3' }\end{array}$ \\
IGF-I & NM 001082478.1 & $\begin{array}{l}\text { Forward 5'-GCACTCTGCTTGCTCACCTTTA-3' } \\
\text { Reverse 5'-TCCGAATGCTGGAGCCATA-3' }\end{array}$ & 150 \\
IGF-IR & NM 052807.2 & Forward 5'-GGTCTCTAAGGCCAGAGGTGGA-3' & 148 \\
& & Reverse 5'-GACGAACTTGTTGGCATTGAGGTA-3' & 122 \\
\hline
\end{tabular}


Billerica, MA, USA). The membrane was incubated overnight at $4{ }^{\circ} \mathrm{C}$ with the monoclonal IGF-I antibody and the monoclonal IGF-IR antibody (Abcam, UK) at a 1:1000 dilution. The secondary antibodies (Zhongshan Golden Bridge Biotechnology, China) were diluted 1:3000 in TBST. The membrane was incubated with secondary antibodies for $1 \mathrm{~h}$ at room temperature. The membranes were then washed and developed.

\section{RNA interference}

We designed the interference vector (Table 2) and four interference sequences to knock down the IGF-I gene (Table 3). We synthesized double-stranded DNA containing interference sequences and ligated them into the lentiviral transfer plasmid. Third passage cells were divided into six groups: a normal control group (non-transfected group), a negative control group (transfected with control siRNA), and 4 siRNA transfection groups (transfected with each of the 4 siRNAs targeted against the IGF-I gene). The cells were transfected with the lentiviral vectors for $48 \mathrm{~h}$. The mRNA expression of target

Table 2. The frame of interference vector.

\begin{tabular}{|c|c|c|c|c|}
\hline Linker & Sense & Loop & Antisense & Terminator \\
\hline CCGG & & CTCGAG & & TTTTTTg \\
\hline ААТT CAAAAAA & & CTCGAG & & \\
\hline
\end{tabular}

Table 3. IGF-1 RNA interference sequence.

\begin{tabular}{cc}
\hline No & Target sequence \\
\hline siRNA1 & 3'-GCACTCTGCTTGCTCACCTTT-5' \\
SiRNA2 & 3'-GCTCTTCAGTTCGTGTGTGGA-5' \\
siRNA3 & 3'-GGCATTGTGGATGAGTGTTGC-5' \\
siRNA4 & 3'-GGAAGTACACTTGAAGAACAC-5' \\
\hline
\end{tabular}

genes in the osteoblasts was tested by RT-PCR.

\section{Combined siRNA and bLF treatment}

Cells were divided into the following groups: a normal control group (non-transfected group); a $10 \mu \mathrm{g} / \mathrm{mL}$ bLF; a negative control group $+10 \mu \mathrm{g} / \mathrm{mL}$ bLF; siRNA transfection group; and an siRNA transfection group $+10 \mu \mathrm{g} / \mathrm{mL}$ bLF. The cells were transfected with the lentiviral vectors for $48 \mathrm{~h}$ and then the culture medium was replaced with medium containing $10 \mu \mathrm{g} / \mathrm{mL}$ bLF for $24 \mathrm{~h}$. Apoptosis was determined by flow cytometry.

\section{Statistical analysis}

The results are reported as the means \pm standard deviation (SD). Data analyses were performed using the Statistical Package for the Social Sciences (SPSS version 16.0) software package. The differences among the groups were determined using a one-way ANOVA model with post-hoc tests. Differences were considered significant if $P<0.05$.

\section{Results \\ Identification of primary cultured rat osteoblasts Cell morphology}

Typical features of osteoblasts were adherent growth and abundant cytoplasm. Cells that were considered to display irregular morphology included triangular, polygonal, and long shuttle-shaped cells (Figure 1A).

\section{Alkaline phosphatase staining}

Blue-violet nuclei were considered positive for alkaline phosphatase. Brown particles were observed within the cytoplasm and some dispersed around the surrounding cells (Figure 1B).

\section{Type-I collagen immunohistochemistry}

In accordance with the defined characteristics of osteoblasts, brown cytoplasmic staining was observed (Figure 1C).

\section{The effects of bLF on the apoptosis of primary osteoblasts}

As shown in Figure 2, both 10 and $100 \mu \mathrm{g} / \mathrm{mL}$ bLF significantly inhibited the apoptosis of primary osteoblasts compared to untreated controls. At a concentration of $10 \mu \mathrm{g} / \mathrm{mL}$, bLF inhibits apoptosis by $70 \%$. We also found that bLF mainly affected early stages of apoptosis and total apoptosis. In bLFtreated groups $(1,10$, and $100 \mu \mathrm{g} / \mathrm{mL})$ total apoptosis was significantly inhibited $(P<0.01)$ compared to the control group. bLF at concentrations of 1,10 , and $100 \mu \mathrm{g} / \mathrm{mL}$ also inhibited early stages of apoptosis, whereas $1000 \mu \mathrm{g} / \mathrm{mL}$ bLF stimulated
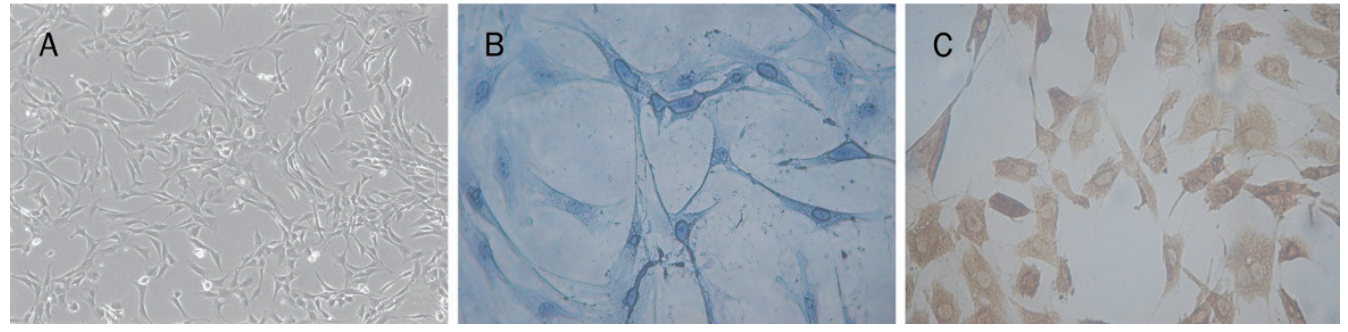

Figure 1. (A) Primary cultured rat osteoblasts were incubated for $48 \mathrm{~h}, \times 200$. (B) Alkaline phosphatase staining, $\times 400$. (C) Type-I collagen immunohistochemistry, $\times 400$. 

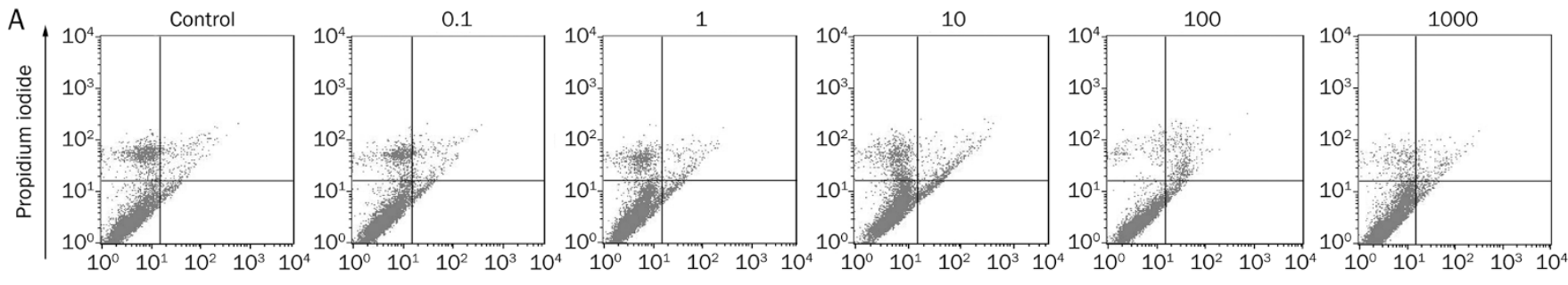

Annexin V-FITC

bLF concentration $(\mu \mathrm{g} / \mathrm{mL})$

B

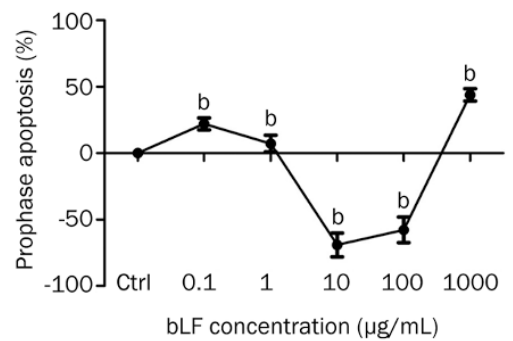

C

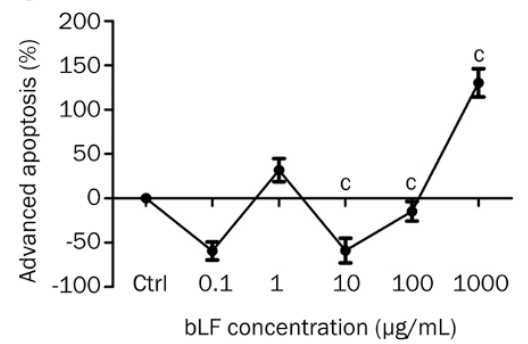

D

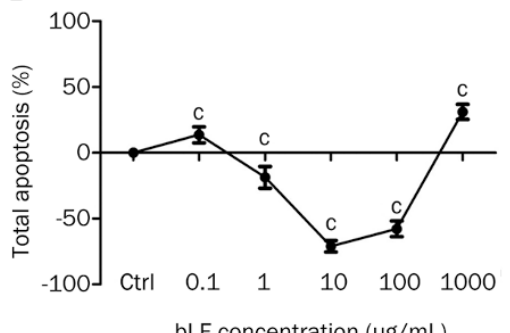

Figure 2. The rate of apoptosis in the different apoptotic phases in response to treatment with varying concentrations of bLF for $24 \mathrm{~h}$. (A) The rate of prophase apoptosis. (B) The rate of advanced apoptosis. (C) The rate of total apoptosis. ${ }^{b} P<0.05,{ }^{c} P<0.01$ compared to the control group. Each experiment was performed in triplicate.

both early stages of apoptosis and total apoptosis. However, varying concentrations of bLF treatment caused no significant alterations in late stages of apoptosis.

\section{The effects of bLF on IGF-I and IGF-IR gene expression}

The addition of bLF to the culture medium increased the level of IGF-I mRNA expression. A significant increase in IGF-I mRNA expression was observed at a bLF concentration of $10 \mu \mathrm{g} / \mathrm{mL}$, while the highest mRNA expression level was observed at a concentration of $100 \mu \mathrm{g} / \mathrm{mL}$ bLF (Figure 3A). Furthermore, addition of bLF to the medium significantly increased the levels of IGF-IR mRNA in a dose-dependent manner. The highest bLF concentration tested $(1000 \mu \mathrm{g} / \mathrm{mL}$ bLF) was the most effective at promoting IGF-IR mRNA expression (Figure 3B).

\section{The effects of bLF on IGF-I and IGF-IR protein expression}

The addition of bLF to the culture medium significantly increased the levels of IGF-I protein in a dose-dependent manner. The highest bLF concentration tested $(1000 \mu \mathrm{g} / \mathrm{mL} \mathrm{bLF})$ was the most effective at promoting IGF-I protein expression (Figure 4A). Moreover, addition of bLF to the culture medium significantly increased the level of IGF-IR protein in a dosedependent manner. The highest bLF concentration tested $(1000 \mu \mathrm{g} / \mathrm{mL})$ was also the most effective at promoting IGF-IR protein expression (Figure 4B).

\section{Optimal selection of siRNAs}

The cells were transfected at a high efficiency (>70\%) with lentiviral vectors that contained one of the 4 targeted siRNAs or the negative siRNA (Figure 5). Based on the outcome of RT-PCR analyses, we confirmed that siRNAs 1-4 all achieved
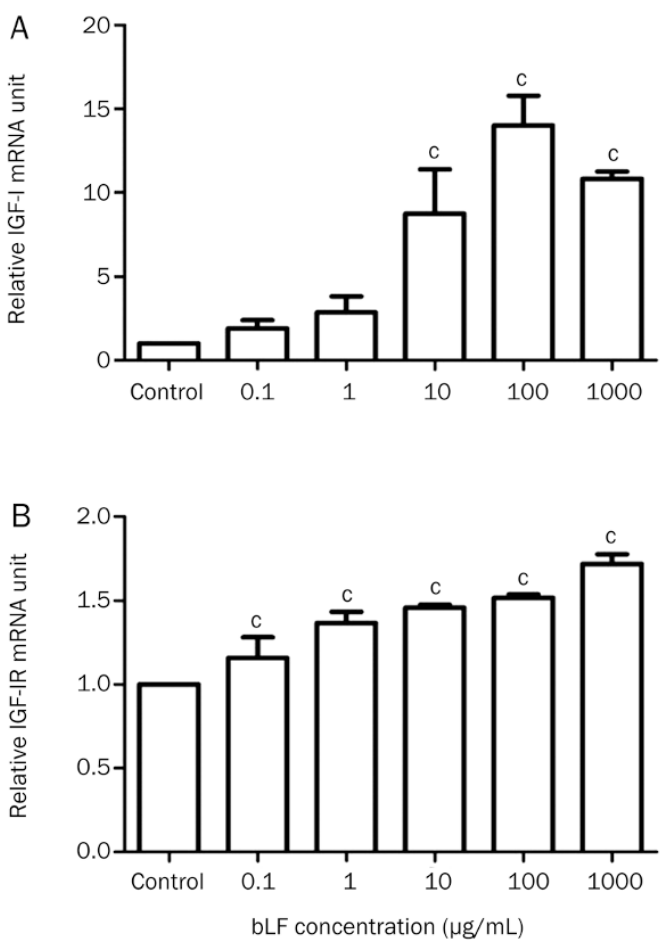

Figure 3. (A) The expression of IGF-1 mRNA in response to treatment with varying concentrations of bLF for $24 \mathrm{~h}$. (B) The expression of IGF$1 \mathrm{R}$ in response to treatment with varying concentrations of bLF for $24 \mathrm{~h}$. ${ }^{\mathrm{c}} P<0.01$ compared to the control group. Each experiment was performed in triplicate.

knockdown of the IGF-I gene compared to the negative siRNA $(P<0.01)$. The most efficient gene silencing sequence was 


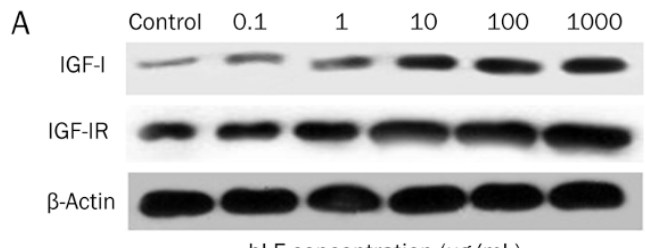

bLF concentration $(\mu \mathrm{g} / \mathrm{mL})$
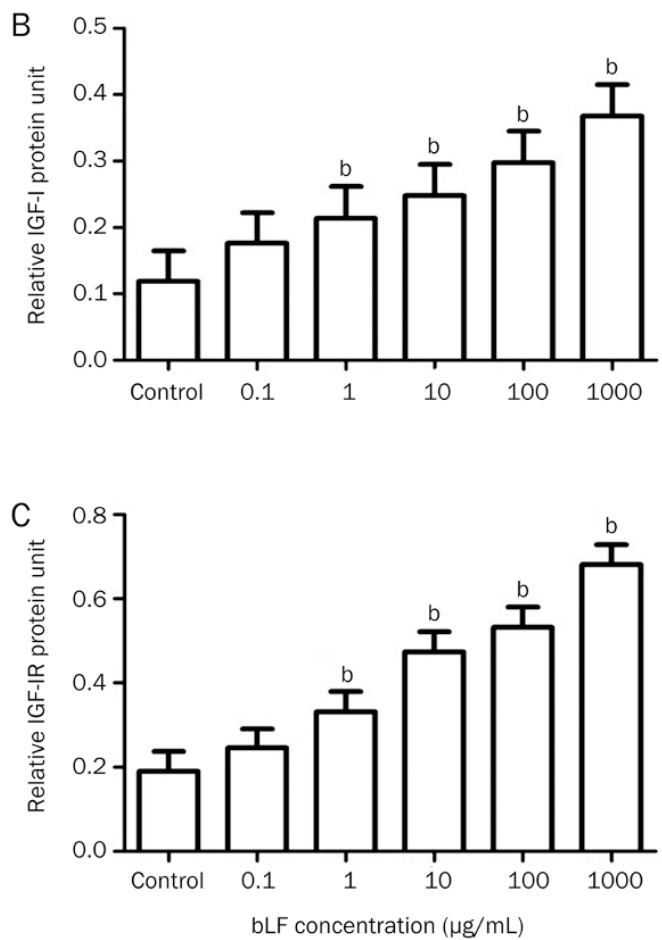

Figure 4. (A) The expression of IGF-1 protein in response to treatment with varying concentrations of bLF for $24 \mathrm{~h}$. (B) The expression of IGF$1 \mathrm{R}$ protein in response to treatment with varying concentrations of bLF for 24 h. ${ }^{b} P<0.05$ compared to the control group. Each experiment was performed in triplicate.

siRNA 1. The negative siRNA sequence had no effect on IGF-I gene expression (Figure 6).

\section{The effects of IGF-I siRNAs on anti-apoptotic action of bLF on rat osteoblasts}

Apoptosis was significantly inhibited $(P<0.01)$ compared to the normal control group in both the $10 \mu \mathrm{g} / \mathrm{mL}$ bLF group and the negative control group $+10 \mu \mathrm{g} / \mathrm{mL}$ bLF. In the siRNA transfection group, the rate of apoptosis increased by $14 \%(P<0.05)$. There were no significant differences in the rate of apoptosis between the siRNA transfection group and the siRNA transfection group $+10 \mu \mathrm{g} / \mathrm{mL}$ bLF $(P>0.05)$. Knockdown of the IGF-I gene in osteoblasts with siRNA markedly increased the osteoblast apoptosis (Figure 7).

\section{Discussion}

The present study found that bLF significantly inhibited osteoblast apoptosis at a concentration of $10 \mu \mathrm{g} / \mathrm{mL}$. Interestingly,
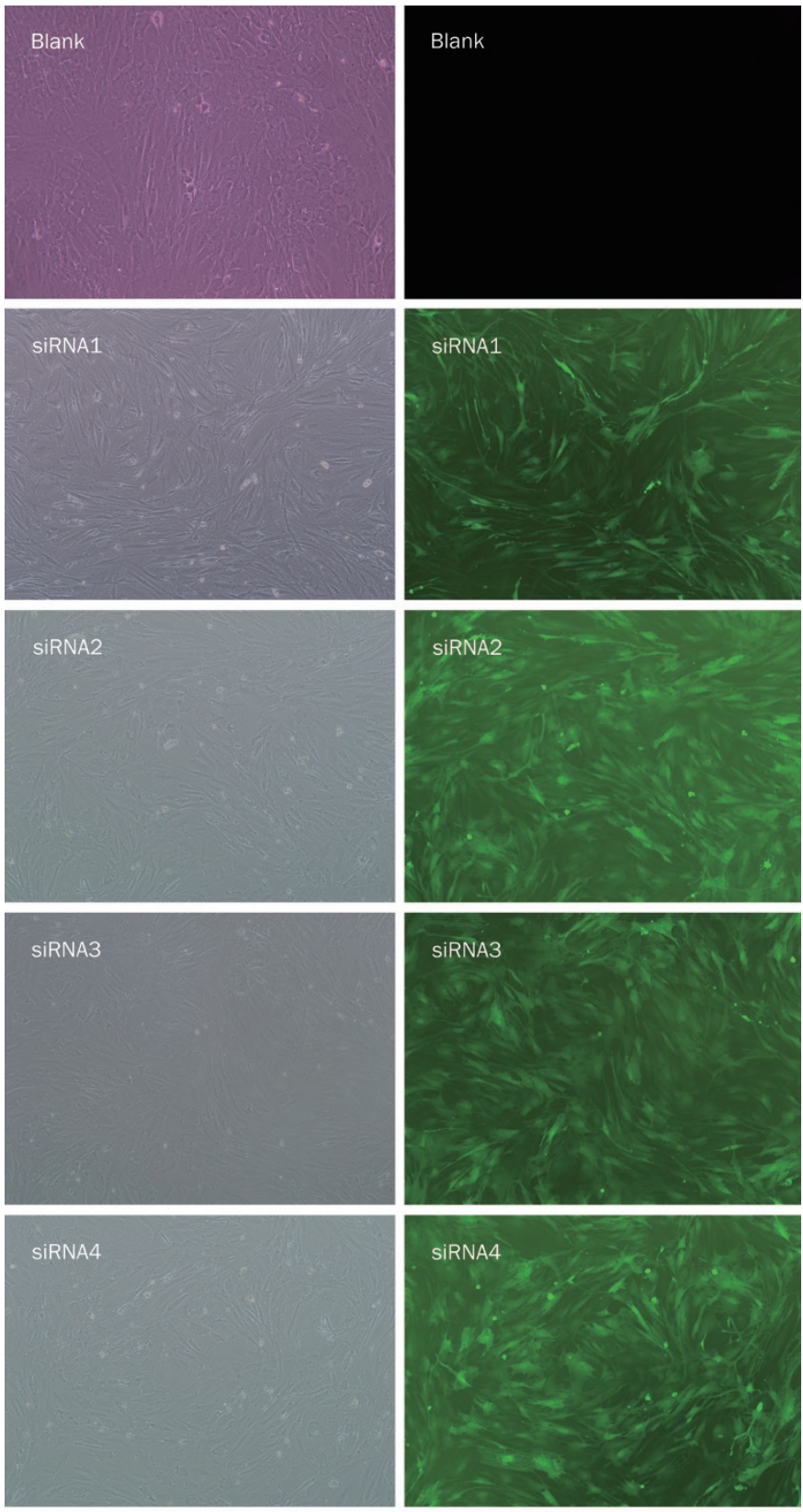

Negative

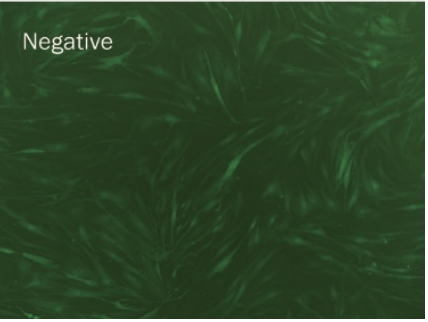

Figure 5. The expression of the green fluorescent protein in osteoblasts. The $\mathrm{MOI}$ was 60 . The rate of transfection was $>70 \%$. The pictures in the left and right column have the same field. The pictures are photographed under normal microscope (left) and the fluorescence microscope (right).

at a concentration of $1000 \mu \mathrm{g} / \mathrm{mL}$, bLF stimulated apoptosis. These results confirmed that bLF has potent anti-apoptotic 


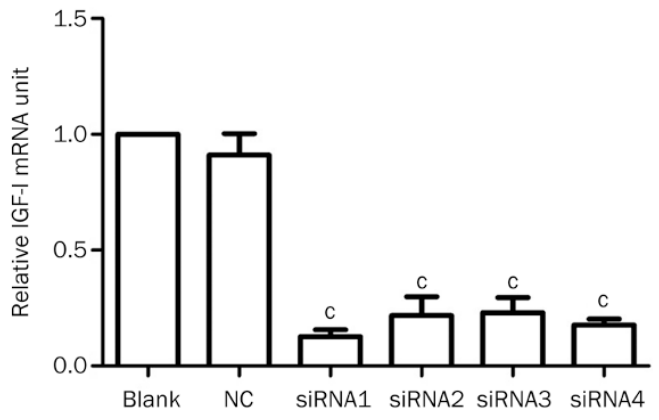

Figure 6. The expression level of IGF-1 mRNA in response to treatment with lentiviral vectors that contained siRNAs 1-4 or the negative siRNA. ${ }^{\mathrm{c}} P<0.01$ compared to the control group. Each experiment was performed in triplicate.

effects on osteoblasts, whereas high concentrations of bLF are toxic to the cells. We also found that bLF promoted the expression of IGF-I and IGF-IR in a dose-dependent manner at concentrations ranging from 1 to $1000 \mu \mathrm{g} / \mathrm{mL}$. Vincent et al ${ }^{[10]}$ reported that cell survival was controlled by the IGF signaling pathway. Therefore, we wanted to elucidate the underlying mechanisms by which bLF inhibits apoptosis of osteoblasts.

Recent studies have demonstrated the importance of osteoblast apoptosis in bone diseases such as glucocorticoidinduced osteoporosis and have shown that agents such as bisphosphonates ${ }^{[11]}$ and estrogen ${ }^{[12]}$ can be used to prevent osteoblast apoptosis. Osteoblast apoptosis is an automatic process of programmed cell death ${ }^{[13]}$ that plays a key role in maintaining the number of osteoblasts. An increase in osteoblast apoptosis is an important cause of low bone mass.
Osteoporosis is a major cause of morbidity and health expenditure in the aging population ${ }^{[14]}$. It is defined as a reduction in bone mass and a change in bone microarchitecture that increases the susceptibility to fracture ${ }^{[15]}$. Bone loss results from an imbalance between the activities of osteoclasts and osteoblasts that leads to an uncoupling of bone resorption and bone formation ${ }^{[16]}$. Current therapies for osteoporosis include calcium, vitamin $\mathrm{D}$, agents that inhibit bone resorption, and other drugs; however, patients are still at risk for developing low bone mass and fractures. An intensive search is underway for the identification of other potential therapeutic compounds that have anabolic effects on bone. Bovine lactoferrin is an iron-binding glycoprotein that belongs to the transferrin family. It is present in breast milk, in epithelial secretions, and in the secondary granules of neutrophils. In healthy subjects, bLF circulates at concentrations of $2 \times 10^{-6}-7 \times 10^{-6} \mathrm{~g} / \mathrm{mL}$. It is a pleiotropic factor with potent antimicrobial and immunomodulatory activities ${ }^{[17]}$. In vitro studies have demonstrated that bLF promotes osteoblast growth both by affecting osteoblast proliferation and by preventing osteoblast apoptosis ${ }^{[18]}$. In this study, we determined the rate of apoptosis at different apoptotic phases in osteoblasts treated with varying concentrations of bLF. Apoptosis was induced by serum deprivation for 24 h. The results showed that bLF mainly affects early stages of apoptosis and total apoptosis in osteoblasts at 1-100 $\mu \mathrm{mol} / \mathrm{L}$, while it has no effects on late stages of apoptosis. In addition, bLF significantly inhibited osteoblast apoptosis at a concentration of $10 \mu \mathrm{g} / \mathrm{mL}$.

Signals from other cells are clearly required for cell survival, cell growth, and proliferation ${ }^{[19]}$. Osteoblasts and oligodendrocytes require specific growth factors such as IGF-I and IGFII when grown in culture ${ }^{[20,21]}$. When deprived of survival fac-
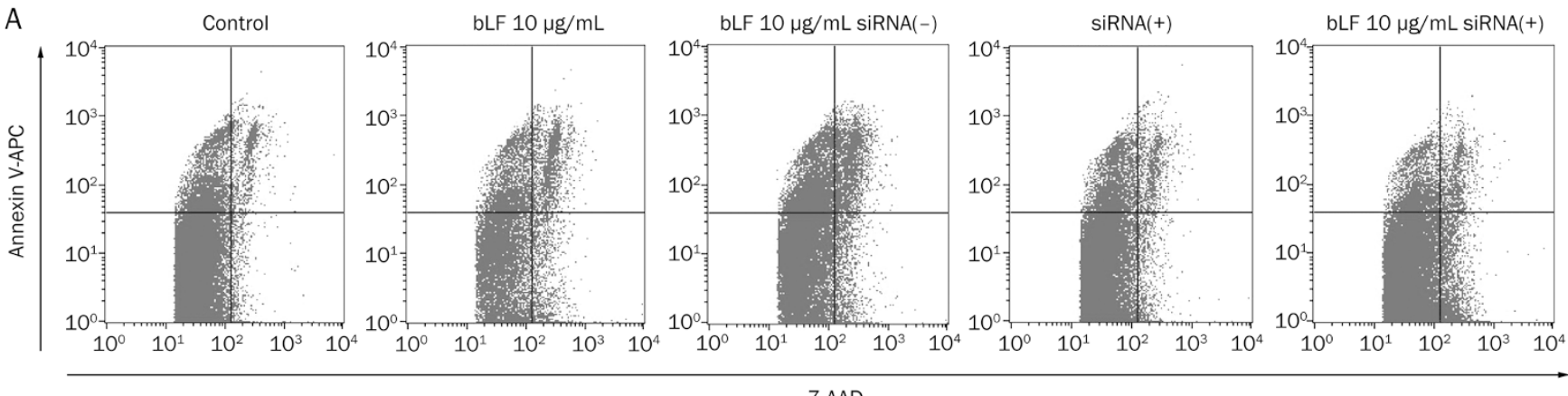

7-AAD

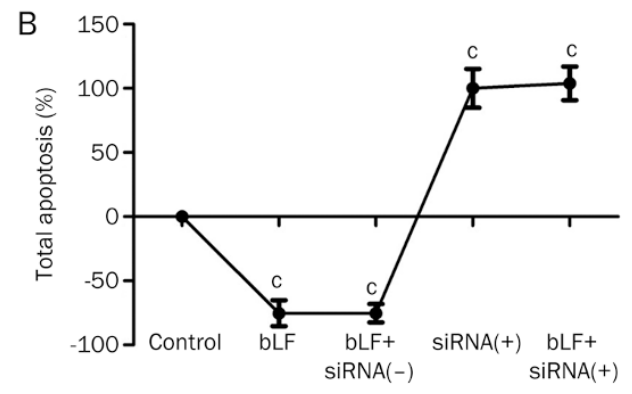

bLF concentration $10 \mu \mathrm{g} / \mathrm{mL}$
Figure 7. The effects of combining bLF and IGF-1 siRNAs on apoptosis. ${ }^{c} P<0.01$ compared to the control group. Each experiment was performed in triplicate. 
tors, these cells appear to activate an intrinsic death program and kill themselves, a process called apoptosis. IGF-I plays an important role in the preservation of bone mass. Recent studies have shown that IGF-I not only functions like insulin by inducing cell proliferation but also plays a crucial role in inhibiting apoptosis. Using neuronal cell lines, Kenchappa ${ }^{[22]}$ found that IGF-I significantly blocked apoptosis induced by tumor necrosis factor- $a$ in vitro. Tumber et $a l^{[23]}$ assessed the effects of neutralizing antibodies on growth factors that are produced by osteoblasts. They found that antibodies against IGF-I and -II were capable of preventing the survival effects of conditioned medium on low density osteoblast cultures whereas antibodies against platelet-derived growth factor (PDGF) and basic fibroblast growth factor (bFGF) had no effect on cell survival. Anti-apoptotic effects that are mediated by the IGF signaling pathway are evident in osteoblasts and other diverse cell types ${ }^{[23]}$. For instance, IGF-IR signaling likely plays an important role in carcinogenesis by inhibiting apoptosis. Thus, IGF-I is important for maintaining proliferation and inhibiting apoptosis in osteoblasts.

Osteoblast apoptosis can be inhibited by bLF. Grey ${ }^{[4]}$ demonstrated that bLF has LRP1-independent anti-apoptotic effects in osteoblasts that do not involve the PI3 kinase or p42/44 MAP kinase signaling pathways. Although bLF activates PI3 kinase-dependent Akt signaling, this effect is neither LRP1-dependent nor required for bLF-induced cell survival. Furthermore, inhibiting the activation of the p42/44 MAP kinase pathway does not abrogate the pro-survival actions of bLF. Nonetheless, the molecular mechanisms by which bLF promotes osteoblast survival remained unknown.

Further investigations into the mechanism of bLF action in osteoblasts are currently underway. Naot used microarrays and low-density arrays to study changes in gene expression induced by bLF in osteoblasts ${ }^{[24]}$. Significant up-regulation of IGF-I and down-regulation of dickkopf homolog 1 (DKK1) mRNAs have been identified in human primary osteoblasts. Similarly, increases in the expression of a number of gene transcripts in rodent osteoblasts, including prostaglandin-endoperoxide synthase 2 (Ptgs2) and nuclear factor of activated $\mathrm{T}$ cells-1 (Nfatc1) ${ }^{[24]}$. The significance of these changes and their ability to promote osteoblast differentiation are currently being investigated. Here we show that the expression of IGF-I and IGF-IR mRNAs are significantly increased in rodent osteoblasts treated with bLF. Treatment of primary osteoblast cells with bLF for $24 \mathrm{~h}$ resulted in a dose-dependent activation of IGF-I and IGF-IR protein expression. Therefore we concluded that bLF might inhibit osteoblast apoptosis by upregulating the expression of IGF-I. Furthermore, IGF-I knockdown promoted osteoblast apoptosis, thereby confirming that bLF inhibits apoptosis via IGF-I.

In conclusion, certain concentrations of lactoferrin can inhibit apoptosis in primary rat osteoblast cultures. The greatest inhibition of osteoblast apoptosis was observed at a concentration of $10 \mu \mathrm{g} / \mathrm{mL}$. Lactoferrin induced the expression of IGF-I and IGF-IR in a dose-dependent manner. Finally, lactoferrin may inhibit osteoblast apoptosis by upregulating the expression of IGF-I.

\section{Acknowledgements}

This study was supported by the National Natural Science Foundation of China (№ 81270968).

\section{Author contribution}

Jian-ming HOU, En-yu CHEN, Shi-chao WEI, and Fan LIN designed the research project. Jian-ming Hou supervised the project. En-yu CHEN performed the in vitro experiments, analyzed the data and wrote the manuscript. Qing-ming LIN, Xuhua LAN, Ying XUE, and Man WU revised the manuscript.

\section{References}

1 García-Montoya IA, Cendón TS, Arévalo-Gallegos S, Rascón-Cruz Q. LF a multiple bioactive protein: an overview. Biochim Biophys Acta 2012; 1820: 226-36.

2 Baker HM, Baker EN. A structural perspective on LF function. Biochem Cell Biol 2012; 90: 320-8.

3 Ward PP, Paz E, Conneely OM. Multifunctional roles of LF: a critical overview. Cell Mol Life Sci 2005; 62: 2540-8.

4 Grey A, Zhu Q, Watson M, Callon K, Cornish J. LF potently inhibits osteoblast apoptosis, via an LRP1-independent pathway. Mol Cell Endocrinol 2006; 251: 96-102.

5 Amini AA, Nair LS. LF: a biologically active molecule for bone regeneration. Curr Med Chem 2011; 18: 1220-9.

6 Cornish J, Naot D. LF as an effector molecule in the skeleton. Biometals 2010; 23: 425-30.

7 Hou JM, Xue Y, Lin QM. Bovine LF improves bone mass and microstructure in ovariectomized rats via OPG/RANKL/RANK pathway. Acta Pharmacol Sin 2012; 33: 1277-84.

8 Cornish J, Callon KE, Naot D, Palmano KP, Banovic T, Bava U, et al. LF is a potent regulator of bone cell activity and increases bone formation in vivo. Endocrinology 2004; 145: 4366-74.

9 Grey A, Banovic T, Zhu Q, Watson M, Callon K, Palmano K, et al. The low-density lipoprotein receptor-related protein 1 is a mitogenic receptor for LF in osteoblastic cells. Mol Endocrinol 2004; 18: 226878.

10 Vincent AM, Feldman EL. Control of cell survival by IGF signaling pathways. Growth Horm IGF Res 2002; 12: 193-7.

11 Plotkin LI, Weinstein RS, Parfitt AM, Roberson PK, Manolagas SC, Bellido T. Prevention of osteocyte and osteoblast apoptosis by bisphosphonates and calcitonin. J Clin Invest 1999; 104: 1363-74.

12 Gohel A, McCarthy MB, Gronowicz G. Estrogen prevents glucocorticoid-induced apoptosis in osteoblasts in vivo and in vitro. Endocrinology 1999; 140: 5339-47.

13 Hill PA, Tumber A. Ceramide-induced cell death/survival in murine osteoblasts. J Endocrinol 2010; 206: 225-33.

14 Cummings SR, Melton LJ. Epidemiology and outcomes of osteoporotic fractures. Lancet 2002; 359: 1761-7.

15 Garnero P, Delmas PD. Evaluation of risk for osteoporosis fractures. In: Bilezikian JP, Raisz LG, Rodan GA, editors. Principles of bone biology. 2nd ed. San Diego, CA: Academic Press; 2002. p 12911301.

16 Ganji MR. Postmenopausal osteoporosis treatment and risk of urinary calculus development. Iran J Kidney Dis 2013; 7: 171-4.

17 Naot D, Grey A, Reid IR, Cornish J. LF - a novel bone growth factor. Clin Med Res 2005; 5: 93-101.

18 Cornish J. LF promotes bone growth. Biometals 2004; 17: 331-5.

19 Mercer WE, Baserga R. The transformation-related p53 protein: a 
potential regulator of cell proliferation. Prog Clin Biol Res 1985; 177 : 171-91.

20 Barres BA, Hart IK, Coles HS, Burne JF, Voyvodic JT, Richardson WD, et al. Cell death and control of cell survival in the oligodendrocyte lineage. Cell 1992; 70: 31-46.

21 Hill PA, Tumber A, Meikle MC. Multiple extracellular signals promote osteoblast survival and apoptosis. Endocrinology 1997; 138: 384958.
22 Kenchappa P, Yadav A, Singh G, Nandana S, Banerjee K. Rescue of TNF-alpha inhibited neuronal cells by IGF-1 involves Akt and c-Jun N-terminal kinases. J Neurosci Res 2004; 76: 466-74.

23 Tumber A, Meikle MC, Hill PA. Autocrine signals promote osteoblast survival in culture. J Endocrinol 2000; 167: 383-90.

24 Cornish J, Naot D. LF as an effector molecule in the skeleton. Biometals 2010; 23: 425-30. 